\title{
Raising the potential of a local market for the reactive power provision by electric vehicles in distribution grids
}

Sousa, Tiago; Hashemi Toghroljerdi, Seyedmostafa; Andersen, Peter Bach

Published in:

IET Generation Transmission and Distribution

Link to article, DOI:

10.1049/iet-gtd.2018.5947

Publication date:

2019

Document Version

Peer reviewed version

Link back to DTU Orbit

Citation (APA):

Sousa, T., Hashemi Toghroljerdi, S., \& Andersen, P. B. (2019). Raising the potential of a local market for the reactive power provision by electric vehicles in distribution grids. IET Generation Transmission and Distribution, 13(12), 2446-2454. https://doi.org/10.1049/iet-gtd.2018.5947

\section{General rights}

Copyright and moral rights for the publications made accessible in the public portal are retained by the authors and/or other copyright owners and it is a condition of accessing publications that users recognise and abide by the legal requirements associated with these rights.

- Users may download and print one copy of any publication from the public portal for the purpose of private study or research.

- You may not further distribute the material or use it for any profit-making activity or commercial gain

- You may freely distribute the URL identifying the publication in the public portal 


\title{
Raising the potential of a local market for the reactive power provision by electric vehicles in distribution grids
}

\author{
Tiago Sousa ${ }^{1 *}$, Seyedmostafa Hashemi ${ }^{1}$, Peter Bach Andersen ${ }^{1}$ \\ ${ }^{1}$ Department of Electrical Engineering, Technical University of Denmark, 2800 Kongens Lyngby, Denmark \\ *tsousa@elektro.dtu.dk
}

\begin{abstract}
The promotion of electric vehicles (EVs), triggered by environmental concerns, can also increase the flexibility of power systems through ancillary services, such as frequency regulation among others. Nevertheless, EV penetration has increased the concern regarding voltage drops in distribution grids. This concern has motivated researchers to examine EV reactive power provision to mitigate such problems. This work proposes a local market perspective to promote EV reactive power provision, enabling distribution system operators (DSOs) to control the voltage level using cost-effective solutions. We propose the extension of a centralized control framework that schedules EV frequency regulation to optimize the reactive power provided by the same EVs. Additionally, we investigate extra power losses in chargers while EVs provide reactive power, and we consider the associated cost in the economic evaluations. A test-case with the IEEE 33-node distribution grid is used to assess the market potential of EV reactive power provision. This new service extends EV penetration in a cost-efficient way without causing voltage problems. The simulation concludes that it is economically feasible to use EVs for reactive local provision with efficient chargers. These outcomes sustain the potential of promoting such new EV services through a proper market in distribution grids.

\section{Nomenclature \\ A. Sets and Indices}

EV Index for electric vehicles

$i, j \quad$ Index for nodes

$L \quad$ Index for loads

$L^{i} \quad$ Set of lines connected to node $i$

$k \quad$ Index for lines

$S P \quad$ Index for External suppliers

$t \quad$ Index for periods

B. Parameters

$\eta_{c} \quad$ Charging efficiency

$B \quad$ Imaginary part in admittance matrix [S]

$c \quad$ Resource cost in period $t$ [m.u./kWh]

$E \quad$ Energy in the EV battery [kWh]

$G \quad$ Real part in admittance matrix [S]

$N \quad$ Number of unit resources

$P F \quad$ Power factor

$S_{L}^{\max } \quad$ Maximum apparent power flow [kVA]

$T \quad$ Total number of periods

\section{$V \quad$ Voltage magnitude [V] \\ $X \quad$ Binary variable}

D. Superscripts and subscripts

$\begin{array}{ll}\text { B,i } & \text { Node abbreviation } \\ \text { BatMax } & \text { Battery capacity } \\ \text { BatMin } & \text { Minimum energy guarantee in period } t \\ \text { Ch } & \text { Charge process } \\ \text { HV / MV } & \text { Transformer that connects the high voltage } \\ \text { and medium voltage } \\ \text { inj } & \text { Initial energy in the EV battery } \\ \text { Load } & \text { Reactive power injected } \\ K & \text { Load } \\ \text { Max } & \text { Line abbreviation } \\ \text { Min } & \text { Upper bound limit } \\ \text { NSD } & \text { Lower bound limit } \\ \text { SP } & \text { External supplier } \\ \text { Stored } & \text { Stored energy in the EV battery } \\ \text { Trip } & \text { Energy consumed in the EV battery during a } \\ & \text { trip }\end{array}$
\end{abstract}

$\bar{y} \quad$ Series admittance of line between two nodes $[\mathrm{S}]$

$\overline{y_{s h}}$

Shunt admittance of line between the node and ground [S]

C. Variables

$\begin{array}{ll}\theta & \text { Voltage angle } \\ E & \text { Energy in the EV battery [kWh] } \\ P & \text { Active power }[\mathrm{kW}] \\ Q & \text { Reactive power }[\mathrm{kVAr}] \\ S & \text { Apparent power }[\mathrm{kVA}] \\ \bar{U} & \text { Voltage in the polar form }[\mathrm{V}]\end{array}$

\section{Introduction}

In recent years, policymakers have assigned significant importance to decreasing the carbon footprint, mainly in power systems and transport sectors [1]. Hence, large investments have been made in distributed energy resources (DERs) [2]. Furthermore, the growing number of electric vehicles (EVs) can benefit the power systems, particularly when using bidirectional chargers and communication through vehicle-to-grid (V2G) technology [3, 4]. Nevertheless, EVs may also create new challenges such as congestion problems or voltage violations, particularly in distribution networks [5]. A distribution system 
operator (DSO) is responsible for guaranteeing that voltage levels remain within an appropriate range. Traditional voltage control is ensured through shunt capacitors and tap changer transformers installed in the distribution grid.

In recent years, the "fit and forget" strategy $[6,7]$ has widely been adopted to deal with DER integration, including EVs, at the distribution level. This strategy resolves issues with DER integration in a centralized manner at the planning stage, without using DERs as part of active management programmes during operation stage. However, such a deterministic approach is not suitable given a high penetration of EVs [8]. Therefore, the recent investigation of active distribution management supports a new role for DSOs $[7,9]$. The key feature assumes the flexibility provided by DERs to support the DSO's operational planning, thereby avoiding new investments in the distribution grid [10]. The aggregator managing an EV fleet is also a relevant actor during such a transition [11]. Voltage control through DER reactive provision is one of the services proposed by the literature $[12,13]$ under this active distribution management.

Recently, interest in EV-provided voltage control has increased because the act of charging the battery causes a voltage drop in the connection node with the grid, which can be mitigated by the same EV with reactive power support. The potential of providing reactive power through an EV charger is addressed in [14-17]. The proof-of-principle of reactive power control via EV chargers is validated in [18]. The studies in $[19,20]$ examine the impact of EVs with different charging strategies on voltage stability. The positive effect of EV reactive control in a real Danish low-voltage distribution grid is shown in [21]. The analytical assessment of reactive support in [22] supports the DSOs planning nextgeneration EV chargers in a low voltage (LV) grid. The authors in [23] analyze the impact of EV reactive support in a LV Flemish distribution grid. A multiagent market-based control mechanism that incorporates voltage support [24] can assign the charging of EVs in a distributed way. Overall, the literature agrees on the EV positive effect via reactive power in voltage support.

Certain literature has focused on the feasibility and impact of EV reactive support in the distribution grid $[16,17$, 19, 21, 23]. Other work [24] paved the way for market-based control of EV voltage support via smart charging. However, a market perspective on EV reactive support requires further investigation. The analysis of the local market potential for EV reactive participation has not been sufficiently explored $[4,25]$. EV owners and respective stakeholders would receive extra incentives created by the local market to participate in the voltage control service. Thus, this paper contributes to the development and assessment of the market potential of EV reactive support. To achieve this objective, the paper examines the provision of EV reactive power under a local market perspective.

The paper implements a centralized control framework to schedule EV fleet reactive participation upon DSO request. We improved the optimization method from [26] to determine the optimal EV reactive participation. Another contribution lies within the developed optimization model that optimally tunes the power factor (PF) for each EV operated by the EV aggregator, although other works assume a fixed PF such as in $[19,21,23]$. Our study takes into account the perspective of different stakeholders (DSO, aggregator, EV user, and charger). More precisely, we assume the economic and technical aspects, e.g. the incorporation of the extra power losses in the charger via simulation. From the charger perspective, it represents the cost of this service to the EV aggregator. Some studies [17, 24, 26] disregarded such costs in their market analysis and business potential for EV reactive provision. Other aspects are also taken into account that will be addressed later in the paper. Overall, our work provides a comprehensive assessment of the impact of new EV reactive market-based service on the grid. Our results show such service solves voltage problems in a cost-efficient way. To the DSO, the solution is economically feasible with efficient chargers. This discussion will be extended later in the paper.

Finally, our centralized control framework can be integrated into a decision-support tool that allocates multiple services to an EV fleet. The platform enables an EV aggregator to participate in multiple services requested by different system operators. For instance, an aggregator can provide the frequency service requested by the transmission system operator (TSO) while injecting the reactive power requested by the DSO. We use the term 'stacked services' to describe the possibility of an EV aggregator's simultaneously providing multiple services.

This paper is structured as follows: after this introductory section, section 2 discusses the potential of a local reactive market for EVs and describes the centralized control framework implemented in this work. Section 3 explains the mathematical formulation of the optimization model behind the centralized control framework. Section 4 presents the case study using an IEEE medium voltage (MV) distribution grid, with realistic EV models and load profiles, and the results are discussed in section 5. Finally, the conclusions are presented in section 6 .

\section{Centralized control framework}

This section is composed of two subsections. Subsection 2.1 discusses the market potential that relies on the EV reactive provision, and subsection 2.2 presents the centralized control framework to support such new service.

\subsection{Market potential of reactive power with EVs}

In the traditional grid operation, power plants were the main contributors to the reactive provision. In the future, the entire power system is expected to be operated with DERs and fossil-fuel power plants will cease to exist or have a residual presence. This leaves room to DERs available at the distribution level to provide such services. The DSOs cannot repeatedly put DERs aside when it comes to solving voltage problems in the grid, as is widely debated in $[6,7]$. The socalled "fit and forget" approach is no longer suitable for future distribution grids with high EV penetration. This leads to a more active management by DSOs to operate the grid using the flexibility provided by EVs, which can postpone new investments in the grid [23]. Such decision-making enables the best use of existing elements, rather than investment in new assets.

A proper cooperation between DSO and EV owners and/or aggregators must be supported by right market-based incentives. It will be witnessed more and more requests by DSOs on having local markets on EV flexibility services [27]. These initial steps foster the potential for having a local market on EV reactive provision. Therefore, a proper tariff scheme has to be designed for this new EV service when EVs 


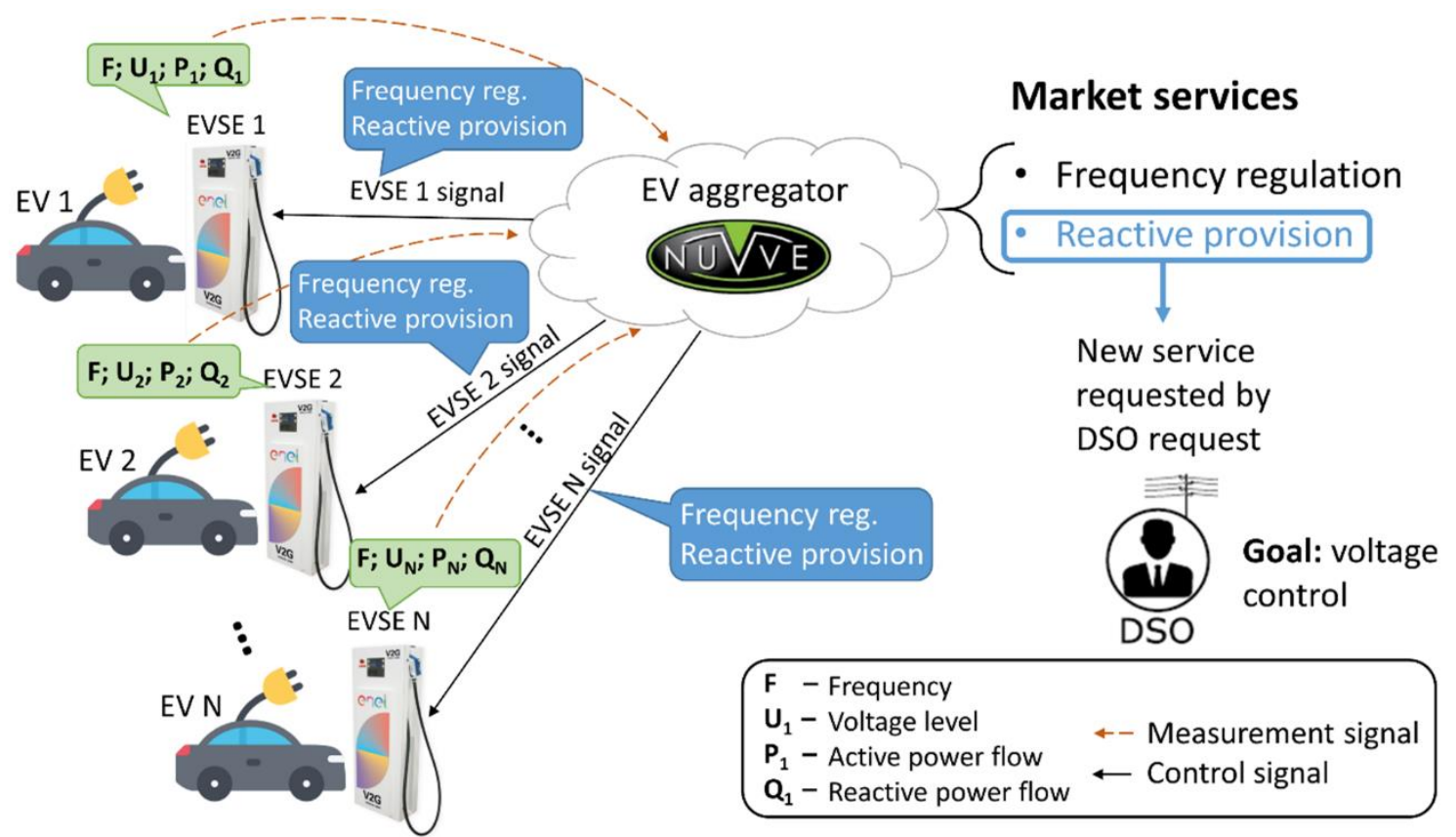

Fig. 1. Architecture of an EV aggregator with the control framework for the reactive power provision

are also charging. The DSO will benefit from this local reactive market because it guarantees a collaborative participation of EVs to address a grid problem that traditional power plants can no longer solve. Such market perspective relies on a bottom-up perspective by letting the EVs cooperate in the DSO operational planning, namely through the reactive provision for voltage problems.

\subsection{Architecture for the EV reactive provision}

This work uses the term EV aggregator to represent an aggregator entity that manages the charging process of EVs. This entity can bid in the energy and reserve markets on behalf of the EV fleet [4]. The centralized control framework for the reactive service is applied to the EV aggregator with a centralized controller [28]. It is presumed an infrastructure with two-way communication between EVs and aggregator. This work assumes that EV trip consumption is known before EV leaves the charging pole. The same is valid for the minimum energy in the battery for emergency trips.

This work is within the scope of PARKER project [29] that investigates the EV fleet participation in the primary frequency regulation and other ancillary services in Denmark. Currently, this project is also collecting field data of Frederiksberg Forsyning EV fleet that provides primary frequency reserve in Eastern Denmark [30]. Within this context, an EV aggregator that is designed by NUVVE [31] has been used to optimally schedule the frequency regulation service provided by the EV fleet. Therefore, our centralized control framework can be included as another EV service to this aggregator. Fig. 1 shows the proposed architecture of the NUVVE aggregator to integrate our centralized control framework. The charging poles are designated as electric vehicle supply equipment (EVSE) in the figure. We also represent the measurement obtained in each EVSE.

First, the DSO requests the EV aggregator to improve the voltage profile in various EVSEs. Then, according to the proposed framework the optimal reactive power of each EVSE is calculated and will be sent to each EVSE to support DSO request. Finally, the EV aggregator will receive the voltage at each EVSE. The measured voltage can be used to tune the reactive power injected in each EVSE. In this way, the aggregator would guarantee the voltage level within DSO required margin. In this paper, we focused on the centralized control framework that optimally determines the reactive signal for each EVSE.

\section{Optimization model}

This section starts by describing the mathematical formulation of the $\mathrm{EV}$ reactive provision problem in subsection 3.1, and the solvers appropriated for this optimization problem are then explained in subsection 3.2.

\subsection{Mathematical formulation}

For this work, we adapted the optimization model proposed in [26] to optimally allocate the EV reactive provision. This optimization model assumes an upstream connection providing the required active and reactive power through external suppliers that define an energy price for providing their active power. They can represent retailers, power plants, wind farms, electricity market pool or bilateral contracts. We also assumed fixed consumption by customers and dumb charging by the EVs. The optimization model calculates the schedule of the external suppliers and reactive power provision for the next 24 hours. A day-ahead forecast of the customers' consumption and the EV trip consumption are considered. The non-linear model of the $\mathrm{AC}$ power flow is also incorporated to check congestions in the MV distribution grid. Since the external suppliers provide the energy required, the objective function minimizes the cost of these resources:

$$
\min F=\sum_{t=1}^{T}\left[\begin{array}{l}
\sum_{S P=1}^{N_{S P}} c_{S P(S P, t)} P_{S P(S P, t)} \\
+\sum_{L=1}^{N_{L}} c_{N S D(L, t)} P_{N S D(L, t)}
\end{array}\right]
$$

where $c_{S P(S P, t)}$ represents the price of active power from supplier $S P$. A penalization is used concerning the nonsupplied demand (NSD), in which the customers are 
remunerated for the non-supplied demand with a specific price $c_{N S D(L, t)}$. This happens when the external suppliers have not enough power to supply all customers' demand.

The minimization of the function $F(1)$ is subject to a set of constraints that will be explained below. First, the model considers an AC power flow [20] to ensure that the results cause no congestion to the grid, such as voltage limits and the line thermal limit. The AC power flow model that is implemented in this paper is adjusted for balanced distribution networks. The active power injected in node $i$ is equal to the active power generation minus the active power demand, and is formulated as

$$
\begin{aligned}
& \sum_{S P=1}^{N_{S P}^{i}} P_{S P(S P, t)}^{i}-\sum_{L=1}^{N_{L}^{i}} P_{\text {Load }(L, t)}^{i}-\sum_{E V=1}^{N_{E V}^{i}} P_{C h(E V, t)}^{i} \\
& =G_{i i} V_{i(t)}^{2}+V_{i(t)} \sum_{j \in L^{i}} V_{j(t)}\left[\begin{array}{c}
G_{i j} \cos \theta_{i j(t)} \\
+B_{i j} \sin \theta_{i j(t)}
\end{array}\right]
\end{aligned}
$$

and the reactive power injected in node $i$ is also considered as

$$
\begin{aligned}
& \sum_{S P=1}^{N_{S P}^{i}} Q_{S P(S P, t)}^{i}-\sum_{L=1}^{N_{L}^{i}} Q_{\operatorname{Load}(L, t)}^{i}-\sum_{E V=1}^{N_{E V}^{i}} Q_{I n j(E V, t)}^{i} \\
& =V_{i(t)} \sum_{j \in L^{i}} V_{j(t)}\left[\begin{array}{c}
G_{i j} \sin \theta_{i j(t)} \\
-B_{i j} \cos \theta_{i j(t)}
\end{array}\right]-B_{i i} V_{i(t)}^{2} \\
& \forall t \in\{1, \ldots, T\} ; i \in\left\{1, \ldots, N_{B}\right\} ; \theta_{i j(t)}=\theta_{i(t)}-\theta_{j(t)}
\end{aligned}
$$

where $\theta_{i j(t)}$ corresponds to the voltage angle difference between nodes $i$ and $j . L^{i}$ is the set of lines connected to node $i$, enabling the calculation of the power flow in each line connected to the same node. $G_{i j}$ and $B_{i j}$ represent the real and imaginary part of the admittance matrix corresponding to the $i$ row and column $j$, respectively. In an AC power flow model, the voltage needs to be between upper and lower limits

$$
\begin{aligned}
& V_{\text {Min }}^{i} \leq V_{i(t)} \leq V_{\text {Max }}^{i} \\
& \theta_{\text {Min }}^{i} \leq \theta_{i(t)} \leq \theta_{\text {Max }}^{i}
\end{aligned}
$$

A slack node is previously selected in the network, and the fixed voltage magnitude and angle are specified for it. The final step for the AC model is to impose an upper limit (line thermal limit) for the power flow from node $i$ to node $j$, and vice versa

$$
\begin{aligned}
& \left|U_{i(t)}\left[y_{i j} U_{i j(t)}+y_{s h_{-} i} U_{i(t)}\right]^{*}\right| \leq S_{L k}^{M a x} \\
& \left|U_{j(t)}\left[y_{i j} U_{j i(t)}+y_{s h j} U_{j(t)}\right]^{*}\right| \leq S_{L k}^{M a x} \\
& \forall t \in\{1, \ldots, T\} ; i, j \in\left\{1, \ldots, N_{B}\right\} \\
& \forall k \in\left\{1, \ldots, N_{K}\right\} ; i \neq j ; U_{i j(t)}=U_{i(t)}-U_{j(t)}
\end{aligned}
$$

where the term $U$ represents the voltage in polar form. The energy from external suppliers goes through HV/MV transformer that connects the distribution grid to the upstream power grid. These transformers have an upper limit $\left(S_{H V / M V}^{M a x}\right)$ that limits the active and reactive power from external suppliers, defined as

$$
\begin{aligned}
& \left(\sum_{S P=1}^{N_{S P}^{i}} P_{S P(S P, t)}^{i}\right)^{2}+\left(\sum_{S P=1}^{N_{S P}^{i}} Q_{S P(S P, t)}^{i}\right)^{2} \leq\left(S_{H V / M V(i)}^{M a x}\right)^{2} \\
& \forall t \in\{1, \ldots, T\} ; i \in\left\{1, \ldots, N_{B}\right\}
\end{aligned}
$$

Regarding the external suppliers, we have a maximum limit for the active and reactive generation:

$$
\begin{aligned}
& P_{S P(S P, t)} \leq P_{\operatorname{Max}(S P, t)} \\
& Q_{S P(S P, t)} \leq Q_{\operatorname{Max}(S P, t)} \\
& \forall t \in\{1, \ldots, T\} ; S P \in\left\{1, \ldots, N_{S P}\right\}
\end{aligned}
$$

In terms of EV constraints, the state of charge (SOC) in each period $t$ is also calculated:

$$
\begin{aligned}
& E_{\text {Stored }(E V, t)}=E_{\text {Stored }(E V, t-1)}-E_{\text {Trip }(E V, t)} \\
& +\eta_{c(E V)} P_{C h(E V, t)} \\
& \forall t \in\{1, \ldots, T\} ; \forall E V \in\left\{1, \ldots, N_{E V}\right\} ; \Delta t=1 \\
& t=1 \rightarrow E_{\text {Stored }(E V, t-1)}=E_{\text {Initial }(E V)}
\end{aligned}
$$

where, $\eta_{c(E V)}$ corresponds to the charging efficiency. $\Delta t$ indicates the period factor used in the optimization model; for instance $\Delta t$ is equal to 1 when we optimize for a period of 1 hour. The optimization model can also be used for other time steps, e.g. 30 minutes, corresponding to $\Delta t=0.5$. Thus, we are able to calculate the SOC of EVs regardless of the time step. $E_{\text {Initial(EV) }}$ represents the energy stored at the beginning of period 1. $E_{\text {Trip }(E V, t)}$ stands for the energy spent in a travel at period $t$, which decreases the SOC. We assume that it contains the daily travel profile (or driving pattern) of each EV user. The $E_{\text {Trip }(E V, t)}$ imposes to the optimization model the required charging for the EV user to travel in the next day.

Furthermore, the SOC in the batteries has a maximum and minimum state of charge given by

$$
\begin{aligned}
& E_{\text {BatMin }(E V, t)} \leq E_{\text {Stored }(E V, t)} \leq E_{\text {BatMax }(E V, t)} \\
& \forall t \in\{1, \ldots, T\} ; \forall E V \in\left\{1, \ldots, N_{E V}\right\}
\end{aligned}
$$

where, $E_{\text {BatMax }(E V)}$ represents the battery capacity. The lower bound $E_{\text {BatMin }(E V, t)}$ works as a minimum reserve at a particular period $t$ requested by the EV user.

The charging power is constrained by a maximum limit, which is formulated as

$$
\begin{aligned}
& P_{C h(E V, t)} \leq P_{\operatorname{Max}(E V, t)} \\
& \forall t \in\{1, \ldots, T\} ; \forall E V \in\left\{1, \ldots, N_{E V}\right\}
\end{aligned}
$$

The reactive power injected by the EV has an upper bound that depends on the charging power and the power factor in the charging point, which is given by

$$
\begin{aligned}
& Q_{\text {Inj }(E V, t)} \leq P_{C h(E V, t)} \tan \left(\cos ^{-1} P F\right) \\
& \forall t \in\{1, \ldots, T\} ; \forall E V \in\left\{1, \ldots, N_{E V}\right\}
\end{aligned}
$$

where, $P F$ is the power factor defined by the DSO that can range from 0 to 1 .

\subsection{Optimization solvers}

This optimization model is classified as a non-linear programming (NLP) problem, due to the nonlinearities introduced by the AC power flow (2) and (3). GAMS software [32] was used to implement the optimization model. Note that a large set of state-of-the-art solvers [33] are available in this platform to solve different classes of the optimization problem (e.g. linear, integer, non-linear programming). In a NLP problem, the local optimal solutions are the obstacles to overcome due to the non-convexities in the search space. Although the commercial solvers in GAMS have algorithms to handle NLP problems, they do not necessarily guarantee the global optimum solution. CONOPT (continuous global optimizer) [34] solver is commonly used to solve NLP problems because presents good performances in NLP problems related to power systems [35]. Thus, the 
authors used CONOPT as optimization solver. This commercial solver is based on generalized reduced gradient method and details on the algorithm can be found in [36].

KNITRO [33] is another well-known commercial solver that uses interior points to solve NLP problems. On the other hand, metaheuristic method [33] is another option to solve NLP problems. Metaheuristic is an iterative process using nature-inspired processes to explore the search space for the optimal solution. Genetic algorithms, particle swarm optimization, tabu search, and simulated annealing are widely used metaheuristic methods. However, they are unable to find the global optimum solution, which is their main disadvantage.

\section{Case study}

This section presents the results of the case study to illustrating the potential of a local market with reactive provision from EVs. This section is divided into five subsections. Subsection 5.1 characterizes the test case used for this work. Subsection 5.2 explains the two strategies for reactive provision in this case study. Subsection 5.3 presents the results of the power flow, while the number of EVs in the grid is increasing. Subsection 5.4 illustrates a comparison in terms of power losses for the two strategies in reactive power support. Subsection 5.5 shows indicators related to the potential of handling reactive provision from a market perspective.

The centralized control framework assumes a balanced 3-phase distribution grid; thereby a single-phase equivalent representation of the grid is considered. The optimization model schedules the reactive power to guarantee that voltage level is between 0.95 and $1.05 \mathrm{pu}$. The optimization model was executed on a computer with two processors Intel ${ }^{\circledR}$ Core $^{\mathrm{TM}}$ i5-6200U $2.40 \mathrm{GHz}$, each one with two cores, $8 \mathrm{~GB}$ of random-access-memory and Windows 10 Enterprise operating system.

\subsection{Case study's characterization}

The case study from [37] is composed by an MV distribution grid with 33 nodes that are connected to the upstream network through a line with 5.5 MVA. This test case contains 218 consumers spread over the grid: 117 domestic consumers, 44 small commerce, 23 medium commerce, 13 large commerce, 8 medium industrial and 13 large industrial consumers. The DG units of the original test case have been discarded because we intend to analyze the impact of EVs in the grid without other DERs. For this case study, the consumers demand is $55 \%$ of the consumption used in reference [37]. This results in a peak demand of 2.1 MW at period 20 and the daily energy consumption is equal to $37 \mathrm{MWh}$.

Fig. 2 depicts the grid topology, where node 0 represents the substation that connects to an upstream network. The energy of an external supplier comes from this substation; assuming an energy price from the wholesale market plus taxes like a retailer that supplies the consumers and EV aggregator. Fig. 3 presents the price assumed for the external supplier. The rest of the data related to this distribution grid is described in [37].

Table 1 shows the EV models used in this case study. We assumed the charger used in the field tests of PARKER project; it is a charger with a bidirectional capability that belongs to ENEL [38].

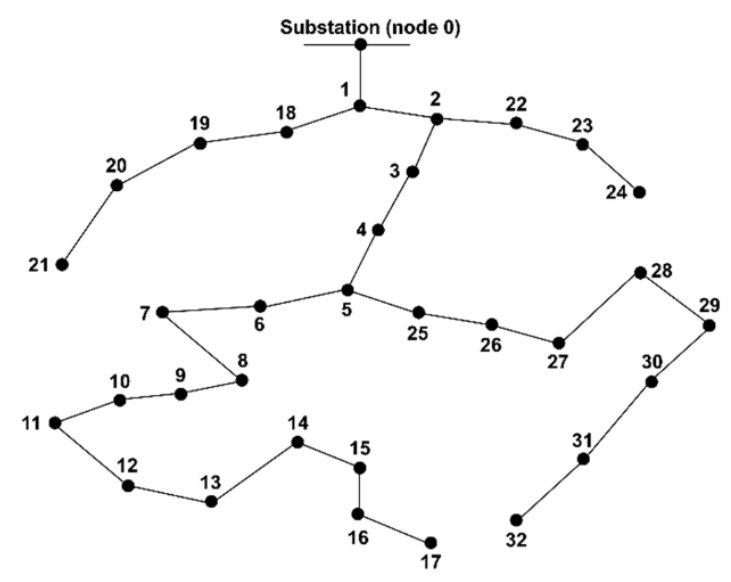

Fig. 2. 33-node distribution grid topology [37]

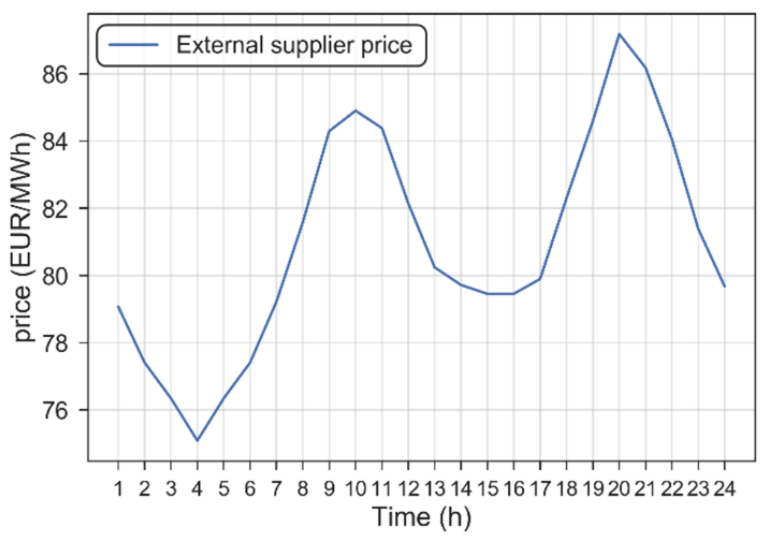

Fig. 3. Energy price of the external supplier price

The study assumes that chargers are available at both public charging spots and homes. EVs are mostly located in the nodes further away from the substation node (node 0), i.e. from nodes 8 to 17 , and nodes 27 to 32 . It is assumed that residential areas are connected to these nodes, which will also have $70 \%$ of EV penetration. For example, 70 out of $100 \mathrm{EVs}$ are located in these nodes. EVs are equally spread over these furthest nodes, thereby $4 \%$ of EV penetration are connected to each one of these nodes. The remaining EV penetration is randomly distributed by the other nodes in this grid. Besides that, we assume that uncertainties related to EV trips are neglected in this study. It is assumed an average trip distance for designing the EV trip in this case study.

\subsection{Reactive strategies}

We define two different reactive strategies to assess the potential of a market perspective in the EV reactive provision. In the first strategy, the reactive power only comes from the substation located in node 0 (see Fig. 2). The TSO guarantees this reactive support as it is done in the traditional approach. Another consists of the distributed reactive support through EVs, while they are charging at the same time. The $\mathrm{PF}$ of the chargers ranges from 1 to 0.9 , which sets the maximum reactive power injected by the EVs (14). The substation also supports the grid with reactive power.

For both strategies, the number of EVs is increased until a point with significant voltage violations. The centralized control framework determines the optimal reactive power for each EV penetration. The goal is to determine the $\mathrm{EV}$ penetration that violates the voltage margin of $0.95 \mathrm{pu}$. Comparing the results from both strategies allows to quantify the potential of $\mathrm{EV}$ reactive provision. 


\subsection{Grid upgrade deferral}

The simulation with the first strategy presents voltage violation for an EV penetration higher than 100 vehicles. Up to this number of EVs, the reactive support from the substation keeps the voltage level of all nodes above $0.95 \mathrm{pu}$. Fig. 4 shows the daily voltage level of four nodes with an EV penetration around 130 vehicles.

Table 1 EVs model data

\begin{tabular}{lcc}
\hline $\begin{array}{l}\text { Vehicle } \\
\text { model }\end{array}$ & $\begin{array}{c}\text { Battery size } \\
(\mathrm{kWh})\end{array}$ & $\begin{array}{c}\text { Energy consumption } \\
(\mathrm{kWh} / \mathrm{km})\end{array}$ \\
\hline Nissan Leaf & 30 & 0.153 \\
Nissan Evalia & 24 & 0.165 \\
Mitsubishi & 12 & 0.165 \\
Outlander & & 0.125 \\
Peugeot iOn & 16 &
\end{tabular}

The minimum voltage limit is represented by a black line to easily identify the periods that violate this requirement. A grey line illustrates the voltage level of 0.955 pu to show when the nodes are close to the minimum voltage limit.

The voltage deviation occurs in the furthest nodes and in the peak periods. If we increase the number of EVs, we have more nodes and periods with voltage deviation, namely in nodes 15 and 32. The 100 vehicles approximately correspond to $20 \%$ penetration of the feeder capacity that

a)

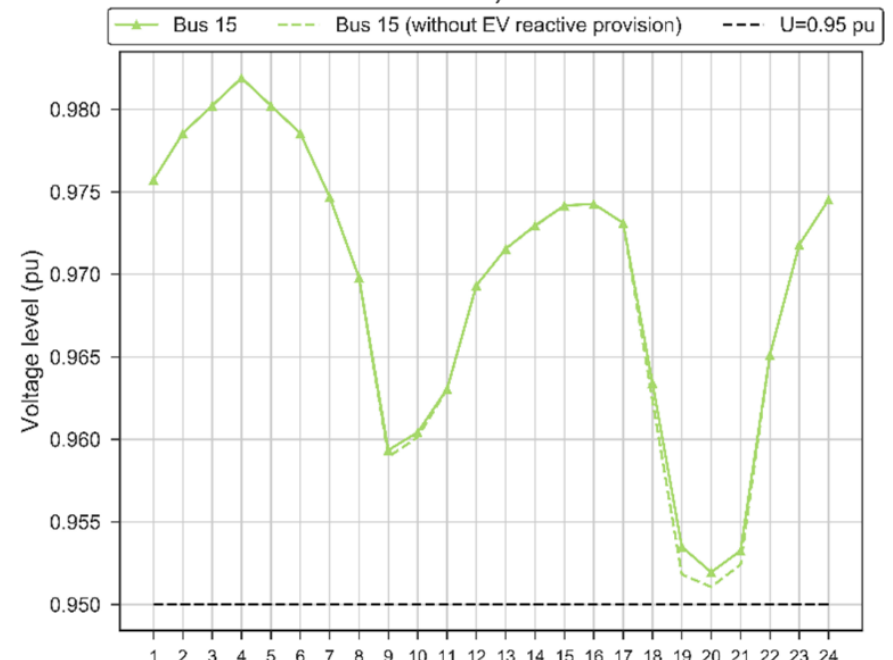

c)

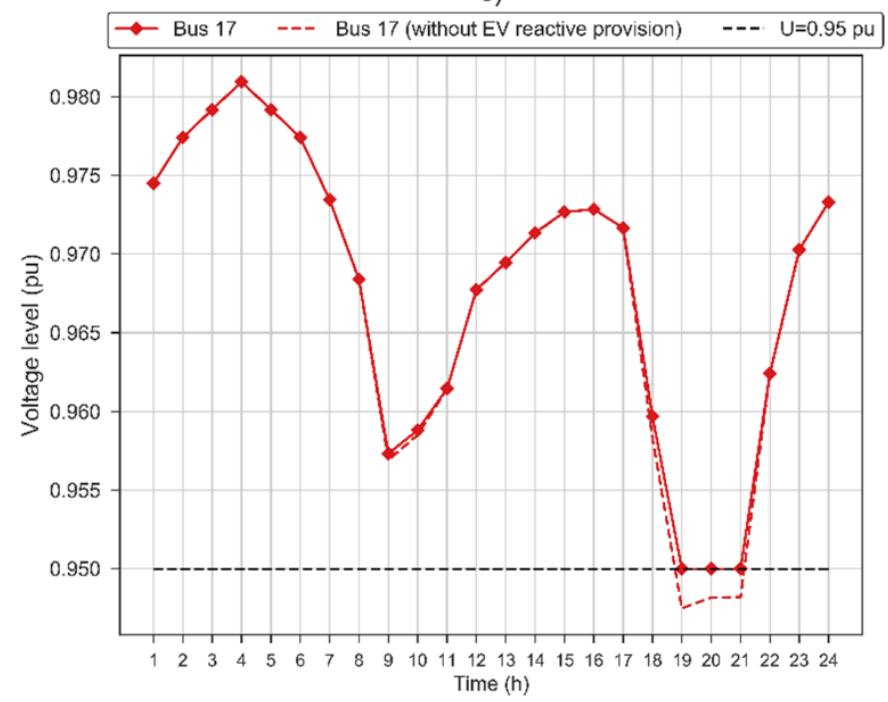

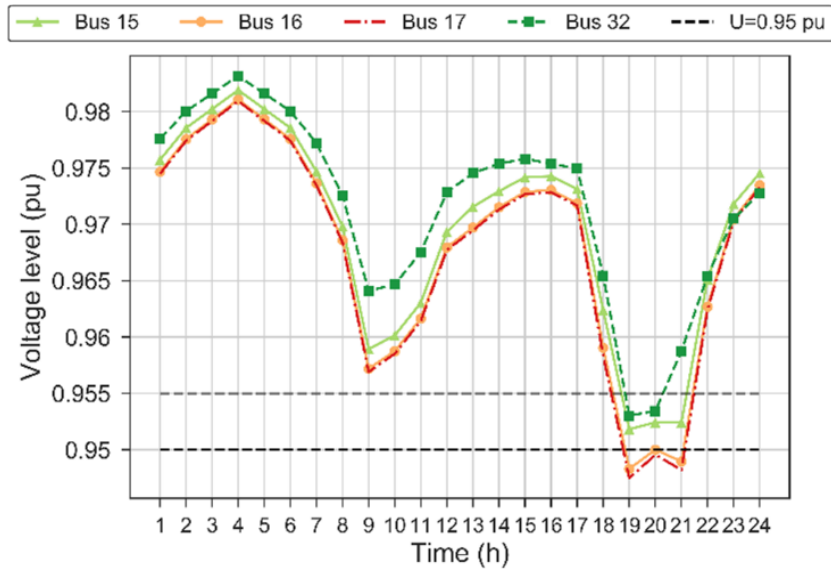

Fig. 4. Voltage level using the first strategy (without EV reactive provision) with 130 cars

connects the substation with the grid (line from node 0 to 1 ). The feeder capacity is equal to 5.5 MVA, and when all 100 EVs are charging we obtain a total charging power of $1 \mathrm{MW}$. We can conclude that raising the feeder consumption above $20 \%$ will cause voltage violation in this distribution grid.

b)

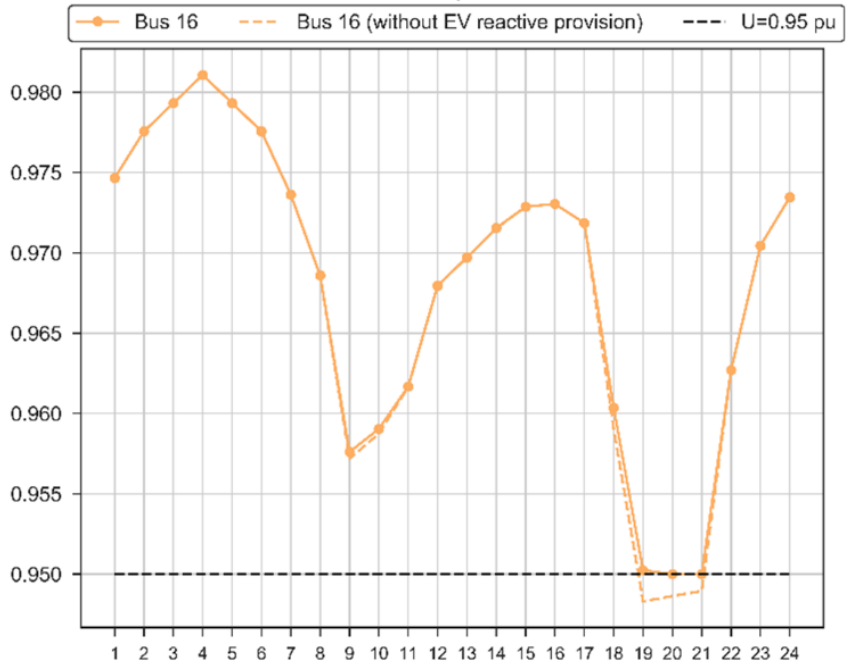

d)

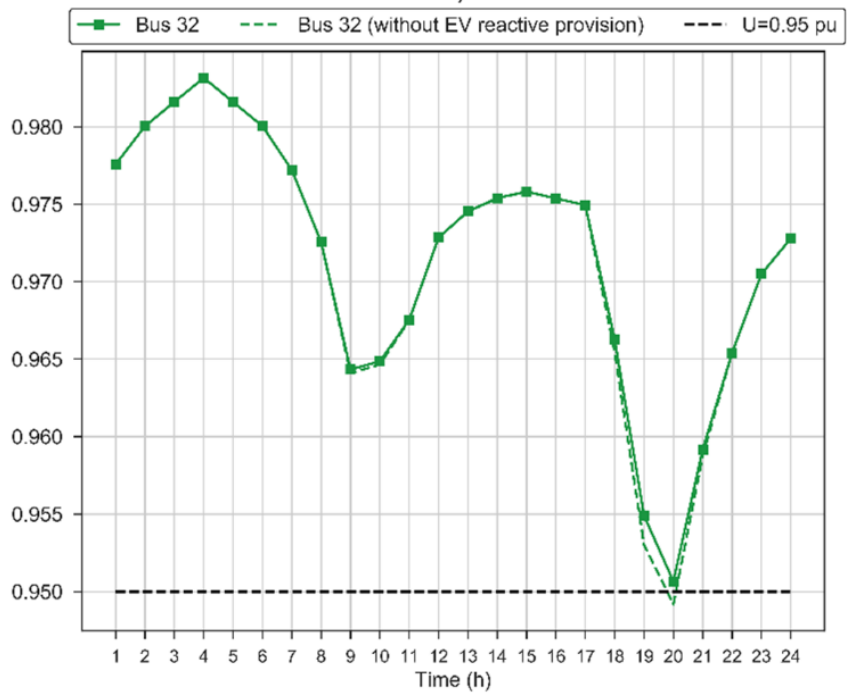

Fig. 5. Voltage level using the EV reactive strategy with 130 cars: a) node 15, b) node 16, c) node 17; d) node 32 


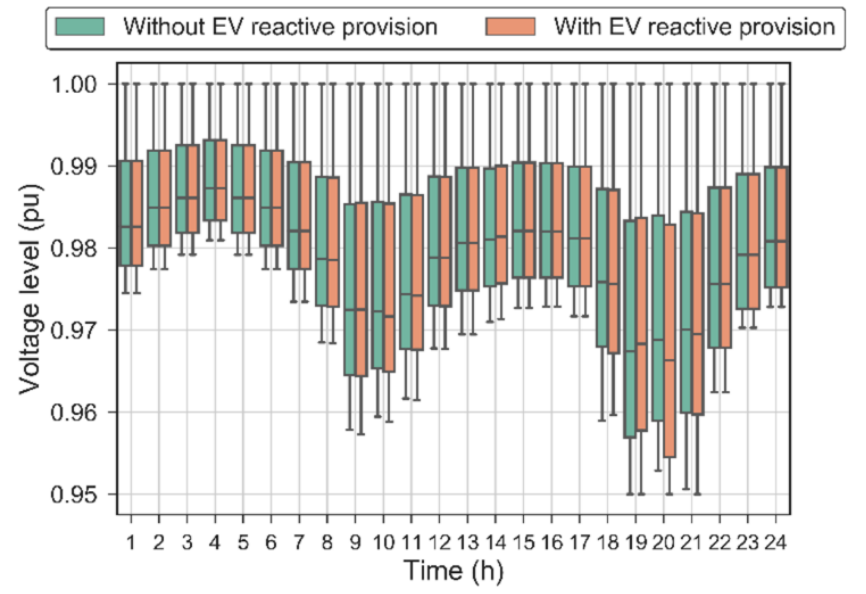

Fig. 6. Variation of voltage profile for the two strategies: green - strategy without EV reactive power and 100 EVs; orange - strategy with EV reactive power and $150 \mathrm{EVS}$

For the simulation with the second strategy, the voltage level is within its boundaries up to 150 vehicles. The optimization model achieved the optimal solution with an average computational time around 7 seconds. Fig. 5 shows the daily voltage level for the same nodes as the previous figure but considering 150 vehicles. The previous voltage deviation in the peak periods disappeared due to the reactive power by the EVs. This new service is able to extend the number of EVs by $50 \%$ (from 100 to 150 vehicles). This corresponds to $30 \%$ penetration of the feeder capacity. This strategy enables the DSO to operate the grid with $10 \%$ more of feeder consumption without having voltage violation occurrences. Fig. 6 shows the impact of both reactive power strategies on the voltage level of all nodes during the period. We compare the simulations without voltage violation - first strategy with $100 \mathrm{EVs}$ and second strategy with $150 \mathrm{EVs}$.

Both strategies present similar voltage profile for a single day, but the second strategy with a higher EV penetration. The voltage level starts to deviate from periods 8 to 12 , which coincides with a peak in the consumers demand. The biggest variations are recorded in the peak periods (i.e. from periods 18 to 21) because both consumers and EVs reach their maximum consumption. Through this figure we can identify the periods with large voltage variations, and how far the average voltage level deviates from the nominal voltage (i.e. $1 \mathrm{pu}$ ). Thus, the periods with necessary EV reactive provision are known.

The EV reactive strategy was also tested for the scenario simulating consumers' demand in a typical summer day. We assumed the $150 \mathrm{EVs}$ with the same charging profile and the aim is to evaluate the effect of consumers' demand on the voltage profile. Fig. 7 shows the average voltage profile for the scenario of summer and winter.

One important finding is that the average voltage in summer is higher than on winter, meaning that the EV reactive strategy is less needed during summer.

Fig. 8 shows the voltage deviation for both strategies when we increase the EV penetration. We simulated up to 500 cars connected in this distribution grid. The voltage deviation is recorded when it drops below $0.95 \mathrm{pu}$. The figure shows the sum of the voltage deviation in all nodes.

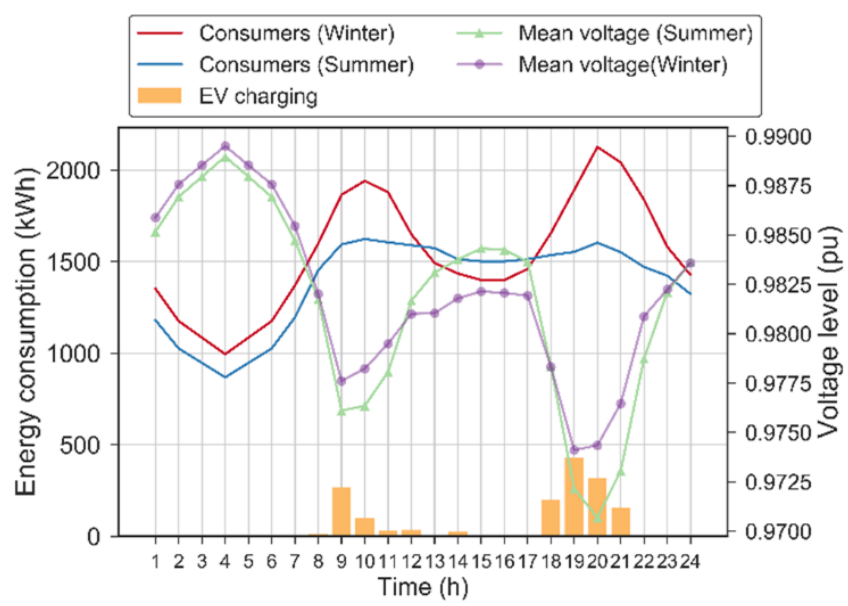

Fig. 7. Average voltage level versus consumers' demandwinter and summer

As expected, the voltage deviation keeps increasing with the increase in EVs penetration. We start having early voltage problems with the first strategy (i.e. more than 100 cars), while the second strategy shifts the voltage problems for a higher EVs penetration (i.e. more than 150 cars). After that, both strategies present voltage deviation, but the second strategy is always able to reach a lower value (when compared with the first one). The distributed allocation of reactive power through EVs also helps to mitigate voltage deviation under scenarios with high EV penetration.

\subsection{Power losses minimization}

So far, we used two strategies to provide reactive support in this grid. The next step aims to assess which strategy suits better the DSO operation. The minimization of the power losses is the goal of the DSO when the grid has no voltage violations. Under this assumption, we compare the two strategies for the same EVs penetration with 100 vehicles, because it is a scenario without voltage violations.

As mentioned before, we assume the characteristics of the charger used in PARKER project. However, there is no available information on the exact value of extra losses by this charger when providing reactive power. Therefore, we simulated 4 different levels of potential extra losses. We define extra losses of $0.5 \%, 1 \%, 2 \%$ and $3 \%$, which reduce the charging efficiency of the charger $\left(\eta_{c(E V)}\right)$. For instance, extra losses of $0.5 \%$ makes the $\eta_{\mathrm{c}(E V)}$ to decrease from $90 \%$ to $89.5 \%$. To compensate for reduction, the optimization model has to increase the charging power for reaching the same final SOC (11). This extra charging will correspond to the extra charger losses for injecting reactive power.

The first strategy without EV reactive provision is the base case for us to compare. For this simulation, the grid registered energy losses per day around $783 \mathrm{kWh}$, while the total charger losses were around $120.5 \mathrm{kWh}$. Then, we run the second strategy with EV reactive provision for each charging efficiency. Table 2 shows the results with the power losses for these simulations.

The column of extra charger losses represents the extra losses registered in the EV chargers when compared with the first strategy without EV reactive provision. The column of grid energy losses corresponds to the decrease in terms of grid energy losses when compared with the first strategy. 


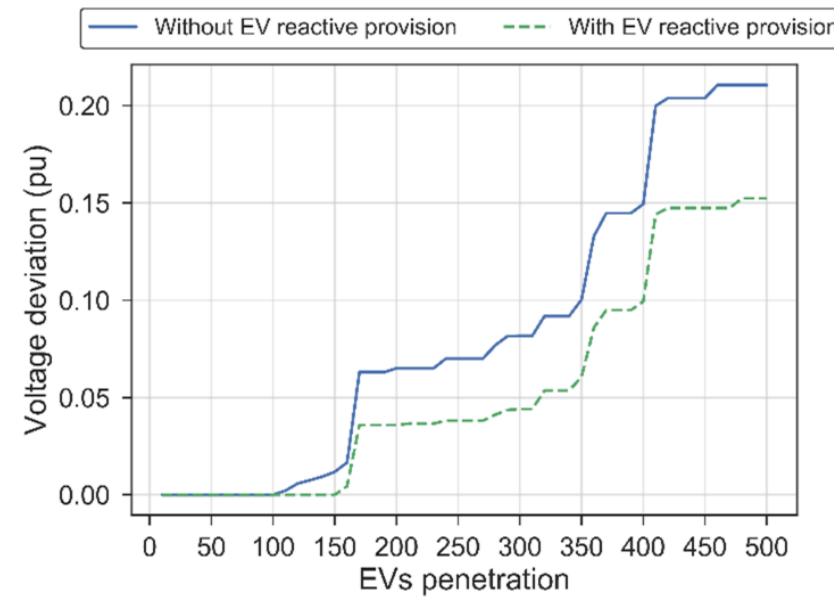

Fig. 8. Voltage deviation per EVs penetration for the two strategies: blue - the strategy without EV reactive power; green - the strategy with EVs reactive power

Table 2 Grid and charger losses for different extra charger losses

\begin{tabular}{lccc}
\hline $\begin{array}{l}\text { Charger } \\
\text { efficiency }\end{array}$ & \multicolumn{2}{c}{ Extra charger losses } & $\begin{array}{c}\text { Grid energy losses } \\
(\mathrm{kWh})\end{array}$ \\
\hline 90 & 0.5 & $\mathrm{kWh}$ & 7.2 \\
& 1 & 6.7 & 6.8 \\
& 2 & 13.5 & 5.9 \\
& 3 & 41.5 & 5.0
\end{tabular}

In the simulation of $0.5 \%$, we have more $6.7 \mathrm{kWh}$ of losses in the chargers. This value must be added to the energy losses achieved in the strategy without $\mathrm{EV}$ reactive provision, i.e. $120.5 \mathrm{kWh}$. Therefore, we have total energy losses of $127.2 \mathrm{kWh}$ for this simulation. The grid energy losses are reduced by $7.2 \mathrm{kWh}$, which results in final energy losses equal to $775.8 \mathrm{kWh}$.

The best simulation is the one with extra charger losses equal to $0.5 \%$ because the increase in the charger losses $(6.7 \mathrm{kWh})$ is smaller than the decrease in the grid energy losses $(7.2 \mathrm{kWh})$. The remaining simulations present more extra charger losses than the decrease in terms of grid energy losses.

\subsection{Reactive power market potential}

As mentioned before, new business models for EVs can arise in the next years by having this local service provision as the backbone. This perspective is appealing to EV owners/aggregator because they can receive an extra revenue by collaborating as an active asset. The reactive energy scheduling for both strategies gives more insight into such potential with EV reactive provision. Fig. 9 depicts the reactive energy scheduled per day. The red line corresponds to the first strategy that the substation is the single provider of reactive power. The blue and green areas respectively represent the reactive provision from substation and EVs in the second strategy.

The EVs are contributing more in the periods with high power demand in the grid. These periods coincide with the time that EVs are connected to charge their batteries. The maximum EV contribution is equal to $35 \%$ of the reactive scheduling. It is achieved in the peak periods, namely during period 19. In fact, the EV contribution is higher than $20 \%$ during the peak periods (i.e. from periods 18 to 20). It is noteworthy that the $\mathrm{EV}$ contribution implies less reactive

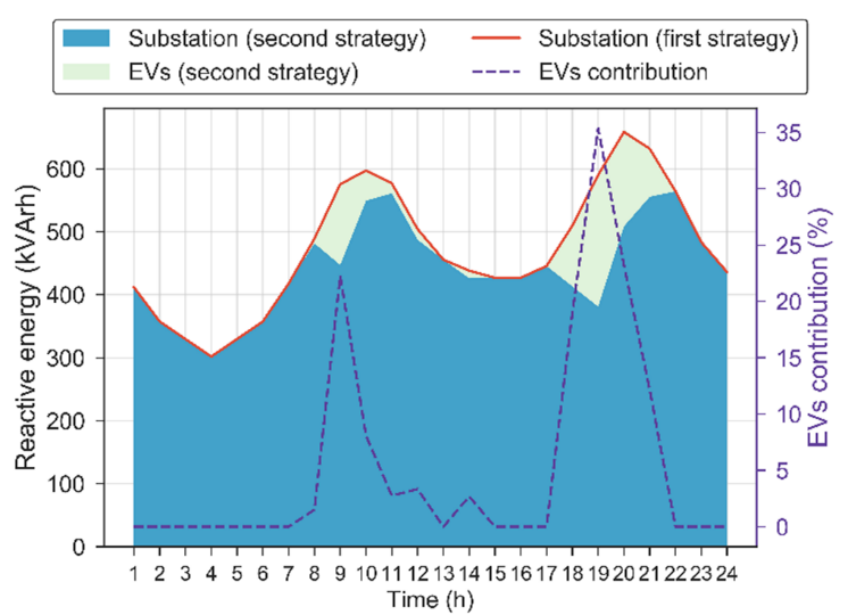

Fig. 9. Reactive scheduling for the two reactive strategies

injection by the substation. Since we have a more distributed reactive provision due to the EV participation, the DSO operation benefits from having power losses decrease.

\section{Discussion of the results}

The discussion will focus on the three topics of the case study: grid upgrade deferral, power losses minimization and the reactive power market. We examine the benefits and implications of EV reactive provision. This assessment of a local reactive market considers the technical and economic aspects of $\mathrm{EV}$ reactive provision.

The case study shows that grid upgrade is delayed through $\mathrm{EV}$ reactive provision. The first reactive strategy (i.e., the traditional approach) shows voltage problems when there are more than 100 cars on the grid (see Fig. 4). On the other hand, the second strategy (i.e., EV reactive provision) extends potential EV penetration to 150 cars (see Fig. 5). Thus, the DSO must invest sooner on corrective measures when adopting the first strategy. An investment plan should be designed to determine the best strategy for upgrading the grid, and, consequently, its cost. This investment plan is outside of the scope of this work because it requires knowing the cost of these devices. Additionally, the investment plan itself is a complex combinatorial optimization problem.

The DSO has the economic benefit of not spending its budget no matter the chosen grid upgrade measure. This creates savings for future necessary investments in the grid. In addition, the DSO gains more time without grid problems that could be used to select the best grid upgrade plan for future voltage problems. After a certain number of EVs, the grid presents voltage problems even with EV reactive provision (see Fig. 8). Therefore, the DSO must invest in the grid, but it might take years to reach more than 150 cars in this case study. Overall, the DSO experiences a twofold benefit: (i) money saved with grid upgrades, and (ii) more time without voltage problems. Furthermore, EV reactive provision was the most effective strategy for minimizing the grid power losses. The best result is reached when we assume low extra losses in the charger (see Table 2). Under this circumstance, the DSO can even use EV reactive provision when there are no voltage problems. Conversely, this solution has the cost of upgrading the EV chargers, which entails to adjusting the control of the power electronic devices inside the charger. This upgrade is not too costly to the EV aggregator or similar players with this kind of infrastructure. 
Thus, we could neglect this cost when compared with the overall economic benefit to the power grid.

Overall, the results of this case study show that the DSO experiences technical and economic benefits in requesting EV reactive provision. There is great value in addressing grid problems with a distributed reactive provision. Therefore, the reactive service could be one of the top services requested by DSOs in the future. We envision DSOs engaging in active distribution management to deploy new services on all types of DERs, particularly EVs [7, 9]. However, the DSO has to find ways of sharing his economic savings with EV owners; otherwise, owners will see it as an unfair use of their assets to solve a problem that is a DSO responsibility. A more market-based approach creates extra incentives for EV collaboration in guaranteeing proper voltage profiles. It also restores the fairness between the DSO and EV owners and aggregators.

This work supports the next generation real-world field applications with commercial chargers and EVs capable of reactive provision. Our centralized control framework optimizes the EV reactive power provision and could be integrated into a future field experiment on this topic. We also intend to raise awareness about the potential of EV reactive provision from a market perspective. Otherwise, there is no true incentive for EV users to participate in grid services. The same resources that introduce voltage problems can also provide reactive service to solve the same problem. A marketbased remuneration can mitigate the drawbacks of EV reactive provision, such as extra losses in the charger (see Table 2). Therefore, the reactive power market is a possibility that is worthy of academic field investigation. Nevertheless, regulatory barriers have to be overcome before the deployment of this new market approach. A defined role of DSOs is required for this new market proposal, specifically because in certain countries the DSO is a non-profit entity.

\section{Conclusions}

The large-scale integration of EVs can stress the operation of MV and LV distribution grids, mainly through voltage problems. The traditional DSO solution is grid reinforcement through new lines and/or transformers. Alternatively, the literature examined the potential for EV reactive power provision without exploring it as a new market-based service. Thus, the main contribution of our study lies in the development and assessment of this market potential of a new EV reactive service. The EVs become active and integrated part of the solution, rather than causing problems through only charging their batteries. This study also expects to pave the way for subsequent real-world field applications.

The proposed methodology can be used to optimally allocate EV aggregator reactive support to solve voltage problems upon DSO request. Our findings show the technical and economic benefits to the involved stakeholders (e.g., DSO, aggregator, EV). First, EV penetration can be increased while the DSO postpones new investments through EV reactive support. In our case study, EV penetration is extended from 100 to $150 \mathrm{EVs}$ without causing voltage problems. The second finding is the trade-off between this new EV service and the charger efficiency. Based on our results, the adoption of this new EV service is economically feasible with efficient chargers because the extra losses in the EV charger are lower than the decrease in grid power losses.

However, future work is necessary to convince EV owners and aggregators to participate in such new service. First, business models capable of supporting a local market on EV reactive power provision should be explored. Further, a proper business model would address the uncertainty related to EV availability. To this end, distributed optimization can add extra value to this investigation. Additionally, test real-world experiments assessing the impact of EV reactive provision should be conducted. EV research projects (such as PARKER) have analysed the potential of EV services on frequency regulation. Similar experiments should be explored in the future.

\section{Acknowledgments}

This work is supported by the Danish EUDP programme through the PARKER project (grant no. 2016-112410). The EV icon used in Fig. 1 was designed by Nikita Golubev from Flaticon (https://www.flaticon.com).

\section{References}

1 Bussar, C., Stöcker, P., Cai, Z., et al.: 'Large-scale integration of renewable energies and impact on storage demand in a European renewable power system of 2050-Sensitivity study', J. Energy Storage, 2016, 6, pp. 1-10.

2 Ellabban, O., Abu-Rub, H., Blaabjerg, F.: 'Renewable energy resources: Current status, future prospects and their enabling technology', Renew. Sustain. Energy Rev., 2014, 39, pp. 748-764.

3 Kempton, W., Tomić, J.: 'Vehicle-to-grid power fundamentals: Calculating capacity and net revenue', J. Power Sources, 2005, 144, (1), pp. 268 279.

$4 \quad \mathrm{Hu}$, J., Morais, H., Sousa, T., Lind, M.: 'Electric vehicle fleet management in smart grids: A review of services, optimization and control aspects', Renew. Sustain. Energy Rev., 2016, 56, pp. 12071226.

5 Yilmaz, M., Krein, P.T.: 'Review of the impact of vehicle-to-grid technologies on distribution systems and utility interfaces', IEEE Trans. Power Electron., 2013, 28, (12), pp. 5673-5689.

6 Abapour, S., Zare, K., Mohammadi-Ivatloo, B.: 'Evaluation of technical risks in distribution network along with distributed generation based on active management', IET Gener. Transm. Distrib., 2014, 8, (4).

7 Li, R., Wang, W., Chen, Z., Jiang, J., Zhang, W.: ‘A Review of Optimal Planning Active Distribution System: Models, Methods, and Future Researches', Energies, 2017, 10, (11).

8 Samimi, A., Kazemi, A.: 'A New Approach to Optimal Allocation of Reactive Power Ancillary Service in Distribution Systems in the Presence of Distributed Energy Resources', Appl. Sci., 2015, 5, (4), pp. 1284-1309.

9 EURELECTRIC: 'Active distribution system management - A key tool for the smooth integration of distributed generation' (2013)

10 Zhao, J., Wang, C., Zhao, B., Lin, F., Zhou, Q., 
Wang, Y.: 'A review of active management for distribution networks: Current status and future development trends', Electr. Power Components Syst., 2014, 42, (3-4), pp. 280-293.

11 San Román, T.G., Momber, I., Abbad, M.R., Sánchez Miralles, Á.: 'Regulatory framework and business models for charging plug-in electric vehicles: Infrastructure, agents, and commercial relationships', Energy Policy, 2011, 39, (10), pp. 6360-6375.

12 Viawan, F.A., Karlsson, D.: 'Voltage and reactive power control in systems with synchronous machine-based distributed generation', IEEE Trans. Power Deliv., 2008, 23, (2), pp. 1079-1087.

13 Camilo, F.M., Castro, R., Almeida, M.E., Fernão Pires, V.: 'Assessment of overvoltage mitigation techniques in low-voltage distribution networks with high penetration of photovoltaic microgeneration', IET Renew. Power Gener., 2018, 12, (6), pp. 649656.

14 Wu, C., Mohsenian-Rad, H., Huang, J., Jatskevich, J.: 'PEV-based combined frequency and voltage regulation for smart grid'. Proc. IEEE PES Innovative Smart Grid Technologies, Washington D.C., USA, January 2012.

15 Zou, N., Qian, L., Li, H.: 'Auxiliary frequency and voltage regulation in microgrid via intelligent electric vehicle charging'. Proc. IEEE International Conference on Smart Grid Communications, Venice, Italy, November 2014, pp. 662-667. Azzouz, M.A., Shaaban, M.F., El-Saadany, E.F.: 'Real-Time Optimal Voltage Regulation for Distribution Networks Incorporating High Penetration of PEVs', IEEE Trans. Power Syst., 2015, 30, (6), pp. 3234-3245.

17 Buja, G., Bertoluzzo, M., Fontana, C.: 'Reactive power compensation capabilities of V2G-enabled electric vehicles', IEEE Trans. Power Electron., 2017, 32, (12), pp. 9447-9459.

18 Yong, J.Y., Ramachandaramurthy, V.K., Tan, K.M., Selvaraj, J.: 'Experimental Validation of a ThreePhase Off-Board Electric Vehicle Charger With New Power Grid Voltage Control', IEEE Trans. Smart Grid, 2018, 9, (4), pp. 2703-2713.

19 Dharmakeerthi, C.H., Mithulananthan, N., Saha, T.K.: 'Impact of electric vehicle fast charging on power system voltage stability', Int. J. Electr. Power Energy Syst., 2014, 57, pp. 241-249.

20 Leemput, N., Geth, F., Van Roy, J., Delnooz, A., Buscher, J., Driesen, J.: 'Impact of electric vehicle on-board single-phase charging strategies on a flemish residential grid', IEEE Trans. Smart Grid, 2014, 5, (4), pp. 1815-1822.

21 Knezović, K., Marinelli, M.: 'Phase-wise enhanced voltage support from electric vehicles in a Danish low-voltage distribution grid', Electr. Power Syst. Res., 2016, 140, pp. 274-283.

Zecchino, A., Marinelli, M.: 'Analytical assessment of voltage support via reactive power from new electric vehicles supply equipment in radial distribution grids with voltage-dependent loads', Int J. Electr. Power Energy Syst., 2018, 97, pp. 17-27.

Leemput, N., Geth, F., Van Roy, J., Büscher, J.,
Driesen, J.: 'Reactive power support in residential LV distribution grids through electric vehicle charging', Sustain. Energy, Grids Networks, 2015, 3, pp. 24-35.

24 Weckx, S., D’Hulst, R., Claessens, B., Driesensam, J.: 'Multiagent charging of electric vehicles respecting distribution transformer loading and voltage limits', IEEE Trans. Smart Grid, 2014, 5, (6), pp. 2857-2867.

25 Ehsani, M., Falahi, M., Lotfifard, S.: 'Vehicle to Grid Services: Potential and Applications', Energies, 2012, 5, (10), pp. 4076-4090.

Sousa, T., Morais, H., Vale, Z., Castro, R.: 'A multiobjective optimization of the active and reactive resource scheduling at a distribution level in a smart grid context', Energy, 2015, 85, pp. 236-250.

27 Knezović, K., Marinelli, M., Zecchino, A., Andersen, P.B., Traeholt, C.: 'Supporting involvement of electric vehicles in distribution grids: Lowering the barriers for a proactive integration', Energy, 2017, 134, pp. 458-468. Andersen, P.B., Poulsen, B., Decker, M., Traeholt, C., Ostergaard, J.: 'Evaluation of a Generic Virtual Power Plant framework using service oriented architecture'. Proc. IEEE 2nd International Power and Energy Conference, Johor Bahru, Malaysia, December 2008, pp. 1212-1217. 'Parker project', https://energiforskning.dk/en/node/8432, accessed April 2018.

30 Arias, N.B., Hashemi, S., Andersen, P.B., Traholt, C., Romero, R.: 'V2G enabled EVs providing frequency containment reserves: Field results'. Proc. IEEE International Conference on Industrial Technology, Lyon, France, February 2018, pp. 1814-1819.

31 'NUVVE', http://nuvve.com/, accessed April 2018.

32 Rosenthal, R.: 'GAMS - A User's Guide'Washington, DC GAMS Dev. Corp., 2008.

33 Keane, A., Ochoa, L.F., Borges, C.L.T., et al.: 'State-of-the-art techniques and challenges ahead for distributed generation planning and optimization', IEEE Trans. Power Syst., 2013, 28, (2), pp. 14931502.

34 Drud, A.: 'GAMS/CONOPT user's notes' (2001).

35 Ghanaatian, M., Lotfifard, S.: 'Control of Flywheel Energy Storage Systems in the Presence of Uncertainties', IEEE Trans. Sustain. Energy, 2019, 10, (1), pp. 36-45.

36 Drud, A.: 'CONOPT-A large-scale GRG code', ORSA J. Comput., 1992, 6, pp. 207-219.

37 Silva, M., Morais, H., Vale, Z.: 'An integrated approach for distributed energy resource short-term scheduling in smart grids considering realistic power system simulation', Energy Convers. Manag., 2012, 64, pp. 273-288.

38 'Enel', https://www.enel.com/, accessed April 2018. 